\title{
Shallow Analysis and the Slingshot Argument
}

\author{
Michael Baumgartner
}

Received: 19 July 2009 / Accepted: 29 March 2010 / Published online: 5 May 2010

(C) Springer Science+Business Media B.V. 2010

\begin{abstract}
According to the standard opinions in the literature, blocking the unacceptable consequences of the notorious slingshot argument requires imposing constraints on the metaphysics of facts or on theories of definite descriptions (or class abstracts). This paper argues that both of these wellknown strategies to rebut the slingshot overshoot the mark. The slingshot, first and foremost, raises the question as to the adequate logical formalization of statements about facts, i.e. of factual contexts. It will be shown that a rigorous application of Quine's maxim of shallow analysis to formalizations of factual contexts paves the way for an account of formalizing such contexts which blocks the slingshot without ramifications for theories of facts or definite descriptions.
\end{abstract}

Keywords Slingshot argument • Logical formalization • Shallow analysis • Factual contexts

\section{Introduction}

Since Church [11], who draws on ideas of Frege [18], a specific type of argument, now mostly known as the slingshot argument, has had considerable influence on various areas in analytic philosophy. The argument has been seen as establishing constraints on the metaphysics of facts and, in consequence, on all kinds of theories drawing on facts, such as theories of truth, correspondence,

\footnotetext{
M. Baumgartner $(\bowtie)$

Department of Philosophy, University of Bern, Länggassstrasse 49a, 3012 Bern, Switzerland e-mail: baumgartner@philo.unibe.ch

URL: www.staff.unibe.ch/baumgart/
} 
truth-making, causation, or explanation. ${ }^{1}$ More concretely, the slingshot yields the paradoxical result that non-extensional (non-truth-functional) sentential connectives linking statements that stand for (or express) facts turn out to be extensional after all, provided that two seemingly unproblematic inference principles are taken to hold for these connectives. Roughly, the first of these principles allows for a truth-preserving substitution of logically equivalent expressions and the second licenses the substitution of co-referring singular terms within contexts governed by a pertaining factual connective. Our informal assessment of the truth conditions of such contexts indeed suggests that both of these substitution principles are correct. Yet, the slingshot argument demonstrates that if these principles are implemented in certain formalisms, the truth-functionality of factual connectives can be inferred, which is an unacceptable result. The consequence is then often drawn that the identity of facts must be spelled out in such a fine-grained way that one of the substitution principles is rendered incorrect after all.

On the face of it, this argument's broad influence is somewhat surprising because the slingshot is an essentially formal argument. Its validity requires a very specific kind of syntax that treats particular formal expressions as primitive referring symbols, viz. expressions governed by iota-operators or class abstraction. Moreover, as we shall see below, informal correlates of the slingshot are invalid arguments. That is, there is a conflict between a formal and an informal assessment of the validity of the slingshot, which conflict lies at the core of the slingshot's force. The formal rooting of the slingshot raises the question as to how a formalism, relative to which expressions as $1 x F x$ or $\{x \mid F x\}$ are referring symbols, can teach us anything about what a suitable theory of facts must look like, how language relates to the world, or what kind of entities stand in causal relations. In areas of philosophical research that are not primarily concerned with formal matters, but with questions as to facts, truth, correspondence, or causation, formalisms are mere tools-very useful tools indeed - that, among other things, enable us to make precise what we mean by informal statements, to explicate why corresponding arguments are valid or invalid, or to resolve semantic ambiguities. Yet, how could a particular syntactic treatment of definite descriptions or class abstracts have any consequences for non-formal matters as the metaphysics of facts?

Indeed, not all authors have drawn consequences from the slingshot that go beyond formal matters. For instance, Gödel [19] essentially reads the argument as a mere confirmation of Russell's theory of descriptions, which treats definite descriptions as quantificational rather than referring expressions (cf. also [36,

\footnotetext{
${ }^{1}$ Slingshots can be taken to impose constraints on the metaphysics of any kind of extralinguistic entities that can be expressed or stated by statements-as facts, states, states of affairs, situations, propositions, and so forth. For simplicity, I subsume all of these entities under the label "facts" in this paper. For a very concise overview over the different uses of the slingshot argument cf. [8], for an in-depth presentation cf. [28, 29] or [37].
} 
291-298]). Analogously, Cummins and Gottlieb [12] interpret the argument to count against systems of set theory that treat class abstracts as primitive, as does e.g. [6]. Instead of taking the slingshot to impose constraints on the metaphysics of facts, these authors hold that the argument gives preference to certain formalisms over others, to certain interpretations of definite descriptions and class abstracts over others.

This paper argues that the primary questions raised by the slingshot argument do neither concern the ontology of facts nor the correct interpretation of definite descriptions or class abstracts. Rather, the slingshot, first and foremost, raises the question as to the proper formal representation of statements about facts, i.e. of statements as

- The fact that Brutus stabbed Caesar is identical to the fact that the son of Servilia Caepionis stabbed Caesar.

- The fact that Brutus stabbed Caesar caused the fact that Caesar died.

For brevity, statements governed by connectives as "The fact that ... is identical to the fact that ..." or "The fact that ... caused the fact that ..." shall be said to constitute factual contexts. The slingshot persuasively demonstrates that factual contexts are not as easily adequately formalized and their truth conditions rendered formally transparent as might have been expected. More precisely, I shall argue that the slingshot argument, in the first instance, prompts the following two questions:

$\left(Q_{1}\right) \quad$ What inference rules must a formal system $\mathcal{F}$ provide such that the truth conditions of factual contexts can be adequately captured by means of $\mathcal{F}$ ?

$\left(Q_{2}\right)$ What criteria regulate the adequate formalization of factual contexts within $\mathcal{F}$ ?

As this paper will show, Quine [32,160]—one of the most eager defenders of slingshots-has introduced a maxim of adequate formalization, the maxim of shallow analysis, a rigorous application of which to statements about facts paves the way for an account of formalizing factual contexts that answers $\left(Q_{1}\right)$ and $\left(Q_{2}\right)$ without any commitments whatsoever to a specific metaphysics of facts or a particular syntactic treatment of definite descriptions.

That is not to say that a metaphysical account of facts that considers facts to be language dependent fine-grained entities or an analysis of definite descriptions according to which the latter are non-referring quantificational expressions do not have implications for answering these questions as well. On the contrary, one of the central upshots of this paper will be that corresponding theories of facts and definite descriptions impose such strong constraints on answers to $\left(Q_{1}\right)$ and $\left(Q_{2}\right)$ that no adequate account of formalizing factual contexts results. Fine-grained fact theories and Russellian analyses of definite descriptions resolve the conflict between the formal and informal assessments of the validity of the slingshot argument only at the price of giving rise to other conflicts between formal and informal validity assessments with respect to arguments involving factual contexts. Blocking the unwanted consequences 
of the slingshot and satisfactorily answering $\left(Q_{1}\right)$ and $\left(Q_{2}\right)$, however, does not force a specific theory of facts or of definite descriptions upon us. A proper theory of adequate formalization for factual contexts avoids the unwanted consequences of the slingshot without ramifications for an analysis of facts or definite descriptions.

\section{The Slingshot}

Before we can look at the details of the slingshot argument, a conceptual distinction must be explicitly introduced that will be of crucial importance for this paper. Formal inferential dependencies among formulas must be distinguished from informal inferential dependencies among natural language statements. While formal dependence among formulas is to be understood relative to a given calculus, two statements are said to be informally dependent if one of them or its negation is judged to necessitate the truth or falsity of the other or its negation without compulsory recourse to any criterion of this necessitation. ${ }^{2}$ Analogously, formal validity of inference schemes, i.e. of formalizations, must be distinguished from informal validity of natural language arguments. While formal validity is uncontroversially defined in a corresponding formal system, there are several different notions of informal validity available (cf. [17]). I shall here simply presuppose the (standard) model-theoretic notion: An argument is informally valid iff under all systematic reinterpretations of its non-logical vocabulary it holds that, whenever the premises are judged to be true, so is the conclusion. That is, informal validity must be understood relative to a given demarcation between logical and non-logical expressions, which, in turn, hinges on a presupposed formalism $\mathbf{L}{ }^{3}$ In order to be able to evaluate the informal validity of arguments by use of suitable formalisms, theories of adequate formalization must guarantee a correspondence between formal and informal validity. In particular, theories of adequate formalization must validate the following validity principle (cf. [24, 30]):

(VP) An argument $S$ is informally valid relative to the demarcation between logical and non-logical expressions given by a formalism $\mathbf{L}$ iff there exists at least one adequate formally valid formalization of $S$ in $\mathbf{L}$.

\footnotetext{
${ }^{2}$ For example, while $p$ and $p \wedge q$ are formally dependent, "Cameron is a mother" and "Cameron is a woman" are informally dependent.

${ }^{3}$ For example, as to propositional logic, only expressions like "and" or "not" count as logical, whereas modal systems additionally treat "necessary" and "possible" as logical expressions. For simplicity, I subsequently only make the relativization of the notion of informal validity to a demarcation between logical and non-logical expressions explicit where it is relevant to the respective context.
} 
Above all, the slingshot argument reveals severe difficulties when it comes to validating (VP) in cases of factual contexts, more specifically, when it comes to reproducing the informal (in)validity of arguments involving factual contexts by means of certain formalisms, viz. formalisms featuring primitive iota-expressions or class abstracts. The following arguments are informally judged to be valid:

1. The fact that Brutus stabbed Caesar is identical to the fact that Brutus stabbed Caesar. Brutus stabbed Caesar if and only if Brutus is the one who stabbed Caesar. Therefore, the fact that Brutus is the one who stabbed Caesar is identical to the fact that Brutus stabbed Caesar.

2. The fact that Brutus stabbed Caesar caused the fact that Caesar died. Brutus is identical to the son of Servilia Caepionis. Therefore, the fact that the son of Servilia Caepionis stabbed Caesar caused the fact that Caesar died.

3. The fact that Brutus stabbed Caesar caused the fact that Caesar died. Brutus stabbed Caesar if and only if Brutus did not not stab Caesar. Brutus is identical to Marcus. Therefore, the fact that Marcus did not not stab Caesar caused the fact that Caesar died.

Relative to all reinterpretations of the non-logical vocabulary embedded in the factual contexts of these arguments it holds that if the premises are judged to be true, so are the conclusions. The informal validity of 1 to 3 suggests that the identity of facts does not depend on which element of a set of logically equivalent expressions or co-referring singular terms is used to state them. Hence, the Principle of Substitutivity for Logical Equivalents (PSLE) and the Principle of Substitutivity for Singular Terms (PSST) seem to hold for factual contexts. PSLE says that if $\phi$ and $\psi$ have the same truth-value relative to all systematic reinterpretations of their non-logical vocabulary, i.e. $\phi=\models \psi$, then, if $\Sigma(\phi)$ is a true sentence containing at least one occurrence of $\phi$, the sentence $\Sigma(\psi)$ is also true, where $\Sigma(\psi)$ results from replacing at least one occurrence of $\phi$ in $\Sigma(\phi)$ by $\psi$. PSST maintains that if two singular terms $\alpha$ and $\beta$ have the same referent, i.e. $\alpha=\beta$, then, if the sentence $\Sigma(\alpha)$ containing at least one occurrence of $\alpha$ is true, $\Sigma(\beta)$, which results from $\Sigma(\alpha)$ by replacing at least one occurrence of $\alpha$ in $\Sigma(\alpha)$ by $\beta$, is true as well.

Factual contexts are informally judged to differ from other non-extensional contexts as "Shamus believes that $F a$ ", i.e. from hyperintensional contexts (cf. [27, 127]): While a closed sentence as $F a$ does not express the language independent fact that the object $a$ is $F$ within a belief context (cf. e.g. [18] or [10]), $F a$ seems to express the fact that the object $a$ is $F$ within a factual context, just as it does outside of that context and just as does any other sentence that is logically equivalent to $F a$. Furthermore, while a singular term as $a$ in "Shamus believes that $F a$ " does not refer to the object $a$, but, say, to the pertinent part or element of Shamus' corresponding belief, the singular term $a$ in "The fact that $F a$ is identical to the fact that $F a$ " indeed refers to the object $a$, or so it seems. In consequence, while the applicability of PSLE and PSST to hyperintensional contexts is informally 
excluded to begin with, the two rules are informally judged to be applicable to factual contexts because logically equivalent expressions and co-referring singular terms seem to function analogously in extensional and factual contexts.

However, if PSLE and PSST are suitably adopted in formalisms that treat expressions of type $\imath x F x$ or $\{x \mid F x\}$ as primitive referring expressions, a number of informally unacceptable consequences are rendered formally derivable. Most of all, it can be shown that factual contexts are extensional, which they most certainly are not. As indicated above and as is well-known from the literature, there are a number of different versions of this argument. They differ with respect to formal means implemented but not with respect to conclusions reached. Two categories of slingshot arguments can be distinguished: While the arguments presented by Church [11], Quine [31,32] or Davidson [13] draw on class abstraction and presume the unrestricted validity of PSLE and PSST in factual contexts, the slingshot as introduced by Gödel [19] and as meticulously reconstructed by Neale $[28,29]$ uses iota-operators and does not presuppose the substitutability of all logical equivalents in factual contexts, but only of socalled Gödelian equivalents, i.e. of expressions as $F a$ and $a=\imath x(x=a \wedge F x)$. None of these differences concerns more than formal detail. Since Gödel's slingshot rests on narrower substitution principles and thus, overall, on weaker assumptions, I join Neale [29] in giving preference to Gödel's argument in the following. ${ }^{4}$ Moreover, as the formal details of this argument have been thoroughly exhibited in the literature, I shall center my subsequent discussion around a simplified and somewhat restricted version of Gödel's argument, which nonetheless suffices to exhibit all the core questions raised by slingshot arguments. For details on the unrestricted Gödelian slingshot the reader is referred to Neale $[28,29]$.

Instead of presupposing that any logical equivalents are substitutable salva veritate (s.v.) in factual contexts, the Gödelian slingshot only assumes that 1-Conversion ( 1 -CONV), which is a standard equivalence transformation for extensional contexts, also holds in factual contexts. Subject to 1 -Conv, an extensional formula $\Sigma(x / \alpha)$, which is the result of replacing every occurrence of $x$ in the formula $\Sigma(x)$ containing at least one occurrence of $x$ by the singular term $\alpha$, can be substituted s.v. by the formula $\alpha=\imath x(x=\alpha \wedge \Sigma(x))$-and vice versa. $\alpha=\imath x(x=\alpha \wedge \Sigma(x))$ is to be read as " $\alpha$ is identical to the unique $x$ which is identical to $\alpha$ and $\Sigma(x)$ " or, more colloquially, as " $\alpha$ is the thing which is $\Sigma$ ". Both relative to a Russellian quantificational understanding of iota-expressions and relative to a suitable referential interpretation, $\Sigma(x / \alpha)$ and $\alpha=\imath x(x=\alpha \wedge \Sigma(x))$ are logically equivalent (cf. [29, Chapters 9, 10]). ${ }^{5}$ If factual contexts are symbolized by @[...], the first crucial inference rule

\footnotetext{
${ }^{4}$ Not all authors agree that Gödel's slingshot is preferable. Sobel [37], for instance, favors the Church-Quine-Davidson variant.

${ }^{5}$ Note that referential treatments of definite descriptions give rise to intricate semantic questions in case of so-called improper descriptions. These complications are sidestepped in the present context. For further details cf. [27, Chapter 3].
} 
implemented in Gödel's slingshot can be specified in terms of @+ı-CoNv (cf. [29, Chapter 9]):

$$
@+\text {-Conv : } \frac{@[\Sigma(x / \alpha)]}{@[\alpha=\imath x(x=\alpha \wedge \Sigma(x))]} \frac{@[\alpha=\imath x(x=\alpha \wedge \Sigma(x))]}{@[\Sigma(x / \alpha)]}
$$

@ $+\imath$-Conv simply states that within factual contexts, expressions of type $\Sigma(x / \alpha)$ and $\alpha=\imath x(x=a \wedge \Sigma(x))$ are substitutable s.v.

The second inference rule needed for the Gödelian slingshot is also borrowed from extensional contexts: Whenever for any two definite descriptions $\imath x \Phi x$ and $\imath x \Psi x: \imath x \Phi x=\imath x \Psi x$, then $\imath x \Phi x$ and $\imath x \Psi x$ are substitutable s.v. ${ }^{6}$ Likewise, whenever for any definite description $1 x \Phi x$ and any name $\alpha$ : $\imath x \Phi x=\alpha$, then $\imath x \Phi x$ and $\alpha$ are substitutable s.v. Neale refers to this rule as -Substitution, or $\imath$-Subs for short. If definite descriptions are understood referentially, 1 -Subs is simply a special case of PSST. Adapting 1 -SubS to factual contexts yields the second inference rule needed for Gödel's slingshot (cf. [29, Chapter 7]):

$$
\begin{aligned}
& @+\imath \text {-SUBS : } \quad \imath x \Phi x=\alpha \quad \alpha=\imath x \Phi x \quad \imath x \Phi x=\imath x \Psi x \\
& \frac{@[\Sigma(1 x \Phi x)]}{@[\Sigma(\alpha)]} \frac{@[\Sigma(\alpha)]}{@[\Sigma(1 x \Phi x)]} \frac{@[\Sigma(1 x \Phi x)]}{@[\Sigma(1 x \Psi x)]}
\end{aligned}
$$

As @+ -CoNv and @+ - -SuBs are mere adaptations of PSLE and PSST to a corresponding iota-formalism and as PSLE and PSST informally appear to be correct for factual contexts, there are prima facie no grounds on which to deny the correctness of @+ı-CoNv and @+ı-Subs. However, the Gödelian slingshot now shows that @+r-CoNv and @+ -SuBs allow for formal inferences that we do not take to be informally valid. To illustrate, consider the following statements:

(a) Obama is a politician.

(b) Obama is married.

(c) The fact that Obama is a politician is identical to the fact that Obama is a politician.

(d) The fact that Obama is married is identical to the fact that Obama is a politician.

Informally, we would say that (a), (b), and (c) are true, while (d) is false, for (a) and (b) state different facts about Obama. (a), (b), and (c) do not informally

\footnotetext{
${ }^{6}$ Whenever possible I omit brackets from iota-expressions. That is, $1 x \Psi x$ corresponds to Russell's $(7 x)(\Psi x)$, cf. [38, Chapter $* 14]$.
} 
imply (d). Yet, provided that@+ı-CoNv and @+ -Subs indeed hold, the following is a correct derivation, where $F$ stands for "... is a politician", $G$ for "... is married", $a$ refers to Obama, and @[...] represents the connective "The fact that ... is identical to the fact that Obama is a politician":

$\begin{array}{llll}\left(\zeta_{1}\right) & 1 & {[1]} & F a \\ 2 & {[2]} & G a \\ 3 & {[3]} & @[F a] \\ 1 & {[4]} & a=\imath x(x=a \wedge F x) \\ 2 & {[5]} & a=\imath x(x=a \wedge G x) \\ 3 & {[6]} & \imath x(x=a \wedge F x)=\imath x(x=a \wedge G x) \\ 3 & {[7]} & @[a=\imath x(x=a \wedge F x)] \\ 1,2,3 & {[8]} & @[a=\imath x(x=a \wedge G x)] \\ 1,2,3 & {[9]} & @[G a]\end{array}$
A
A
$\mathrm{A}$
$1,1-\mathrm{CONV}$
2, 1 -CONV
$4,5,1-$ SuBs
3 , @ + - -Conv
$6,7, @+\imath-$ Subs
$8, @+\imath-\mathrm{CoNv}$

$\left(\zeta_{1}\right)$ shows that, if @+ı-CoNv and @+ı-SuBs indeed guarantee the transition from truths to truths, the expressions in lines [1], [2], and [3] formally imply [9]. As [1], [2], [3], and [9] are the formalizations of (a), (b), (c), (d), respectively, $\left(\zeta_{1}\right)$ can be seen to formally exclude that (a) to (c) are true and (d) false, because, as to @+ı-CoNv and @+ı-SuBs, any fact about Obama is identical to any fact about Obama.

The primary problem the slingshot argument reveals is that there exists a conflict between our informal assessment of the inferential (in)dependencies among (a) to (d) and our (attempted) formal representation of these (in)dependencies within a corresponding iota-formalism-call this the slingshot conflict for short. Such a conflict, trivially, can be resolved in one of two ways: (I) Either we take the formal result reached above to force us-by virtue of (VP) - to revise our assessment of the informal (in)dependencies among (a) to (d), or (II) we insist that, notwithstanding the formal result, (a) to (c) can be true, while (d) is false and, hence, that something has gone wrong in the application of our formal apparatus to these statements. Only very few authors, as e.g. [13], even consider resolving the conflict along the lines of (I). To actually question our informal understanding of statements (a) to (d) in light of a formal argument as $\left(\zeta_{1}\right)$ is simply self-defeating. We are as certain as we can possibly get when it comes to assessing inferential (in)dependencies among colloquial statements that the truth of (a) to (c) in no way necessitates the truth of (d). If, in the end, that should turn out to be a false assessment, we would have to conclude that prior to being presented with the slingshot argument we radically misunderstood statements involving factual contexts. Yet, having a reliable understanding of natural language statements is a necessary precondition of being able to adequately formalize these statements. In turn, being reasonably certain that the formalizations involved in the slingshot are adequate, is an essential precondition of drawing any kind of consequences- 
be they metaphysical or formal-from the argument. ${ }^{7}$ Therefore, reaction (I) directly undermines itself.

In consequence, (II) is the only viable manner to resolve the slingshot conflict. Apparently something has gone wrong when we applied our formal tools to render the informal (in)dependencies among (a) to (d) formally transparent. Yet, it is far from obvious where exactly the error has occurred.

\section{Blocking the Slingshot with Metaphysics or Russell}

On the face of it, there are two conceivable sources of error: Either, at least one of the lines [1], [2], [3], and [9] in $\left(\zeta_{1}\right)$ does not feature an adequate formal representation of the corresponding statements (a), (b), (c), and (d), or, contrary to first appearances, at least one of the inference rules @ $+\imath$-Conv and @ $+\imath$-SuBs is incorrect after all. The formalizations contained in [1] to [3] and [9] are utterly simple. They each identify a predicate and a singular term, and two of them additionally feature a sentential connective. All of these elements, and no others, are also contained in the corresponding natural language statements. In consequence, if [1] to [3] and [9] are verbalized along the lines of the provided interpretation, the formalized statements are recovered in their exact natural language form. There is a widely accepted and used maxim in the literature on logical formalization subject to which formalizations that successfully pass this so-called verbalization test are adequate (cf. e.g. [9, Chapter 10], or [36, 64-67]). Accordingly, all authors opting for strategy (II) vis-à-vis the slingshot conflict have localized its source in $@+\imath$-Conv or in @+ı-SuBs (or analogous rules for class abstracts), and not in the formalizations.

Opinions (of course) diverge as to which of these rules to reject and as to why the rejected rule is incorrect. Three opinion groups can be distinguished. The first group-call it $\mathcal{A}$-rejects @ + -Conv (or an analogous rule for class abstracts) and, since @ $+\imath$-Conv is a special case of PSLE, this group also denies the correctness of PSLE for factual contexts. ${ }^{8}$ The second groupgroup B-rejects @+ reads the latter as a iota-version of PSST, which, accordingly is then considered an incorrect rule for factual contexts as well. ${ }^{9}$ Finally, the third group $-\mathcal{C}-$ also

\footnotetext{
${ }^{7}$ For more details on the interplay between informal and formal validity assessments cf. [4] and [3]. ${ }^{8}$ Cf. e.g. $[1,2,14,23,39]$. In fact, all of these authors focus on versions of the slingshot that draw on class abstraction, and, thus, do not literally reject @ $+\imath$-Conv but the corresponding rule for class abstracts.

${ }^{9}$ Cf. e.g. $[25,26]$ or [5].
} 
rejects@+ı-SuBs (or an analogous rule for class abstracts), but does not take that rule to be an adaptation of PSST to a iota-formalism, because definite descriptions (or class abstracts) are not considered to be referring expressions by the authors in $\mathcal{C} .{ }^{10}$

If formal systems used to reproduce factual contexts do not provide inference rules allowing for substitutions of logical equivalents or co-referring definite descriptions and proper names, not all of the arguments 1,2, and 3 can be proven to be valid by formal means. To avert a violation of (VP), authors in groups $\mathcal{A}$ and $\mathcal{B}$ normally read the impossibility to formalize arguments as 1,2 , and 3 by formally valid inference schemes as evidence to the effect that corresponding arguments are in fact invalid. Rejecting the validity of such arguments is then backed up with theories stipulating a fine-grained ontological makeup of facts that, in one way or another, renders the identity of facts sensitive to the particular linguistic expressions chosen to state them. That is, even though arguments as 1,2, or 3 seem to be informally valid, which suggests that facts are-at least in some circumstances—not multiplied by redescribing them by use of different logically equivalent expressions or of co-referring singular terms, representatives of $\mathcal{A}$ and $\mathcal{B}$ read the slingshot argument as evidence to the contrary. ${ }^{11}$ Plainly, this line of reasoning indeed blocks the slingshot. Yet, groups $\mathcal{A}$ and $\mathcal{B}$ are ready to pay a high theoretical price. For analogously to authors opting for strategy (I), members of $\mathcal{A}$ and $\mathcal{B}$ significantly revise assessments of the informal dependencies among statements featuring factual contexts in light of the slingshot argument. That is, they take a formal argument to show that factual contexts as in 1,2, or 3 had been misunderstood prior to the development of that argument. As shall be substantiated in the second half of this paper, a proper account of formalizing factual contexts validates (VP) by, on the one hand, blocking the slingshot argument and by, on the other, formally reproducing the validity of arguments as 1,2 , or 3 .

Representatives of group $\mathcal{C}$, in turn, do not justify their rejection of @ + $\imath$-SuBs along metaphysical lines, but by drawing on Russell's theory of descriptions. As is well known, according to Russell, definite descriptions are incomplete non-primitive symbols. They never occur in isolation, but only in broader sentential contexts, where, on Russell's account, they get a quantificational and not a referential interpretation. ${ }^{12}$ Subject to this theory, which is formally condensed in the famous definition $* 14.01$ of the Principia Mathematica, an identity statement as the one in line [4] of $\left(\zeta_{1}\right)$ is a mere

\footnotetext{
${ }^{10}$ Cf. e.g. [12, 19]. Cf. also [29] for a detailed reconstruction of this line of reasoning.

${ }^{11}$ Of course, the different metaphysical accounts of facts offered by the authors in these first two groups deviate significantly. All that matters for our purposes is that these theories all entail the incorrectness of one of the inference rules implemented in the slingshot.

${ }^{12}$ Cf. e.g. [35, 244-246, 253-254] or [29, 167].
} 
shorthand for the uniquely existential sentence (1), to which PSST cannot be applied.

$$
\exists x(\forall y(y=a \wedge F y \leftrightarrow y=x) \wedge a=x)
$$

That is, representatives of group $\mathcal{C}$ endorse PSST for factual contexts and reject @ $+\imath$-Subs, because the latter treats definitive descriptions as primitive referring expressions which, in fact, they are not. The slingshot is, thus, interpreted as additional evidence for the adequacy of Russell's theory of descriptions.

Before we can look at the merits of this Russellian path around the slingshot, one crucial qualification is called for. Even though Whitehead and Russell [38] do not literally regard definite descriptions as referring expressions, in $* 14.15$ and $* 14.16$ they nonetheless show that, in extensional contexts, expressions of type $\imath x \Phi x$ can be treated as if they were singular terms. They demonstrate that the transitions rendered possible by 1 -SuBS are in fact derivable as theorems in standard first-order logic. 1 -SuBs can, hence, be introduced as a derived rule of inference for quantificationally understood definite descriptions that appear in extensional contexts. That is, rejecting @ $+\imath$-SuBs based on a non-referential interpretation of definite descriptions does only half the work required to block the slingshot. It must moreover be established that the iota-expressions involved in Gödel's argument appear in non-extensional contexts. For if that is not guaranteed, as we shall see shortly, the slingshot can be run based only on @ + $\imath$-CoNv and standard inference rules of first-order logic (including PSST), i.e. even without @+ + -SuBs.

As is well known, a quantificational account of definite descriptions may give rise to ambiguities of scope. A definite description contained in a factual context as @ $[\Phi, x \Psi x]$ can be given a narrow scope reading as expressed in $(n$ $@ 1 x)$ or a wide scope reading as stated in $(w-@ i x) .{ }^{13}$

$$
\begin{array}{ll}
@[\Phi\urcorner x \Psi x] \dashv \models[\exists x(\forall y(\Psi y \leftrightarrow y=x) \wedge \Phi x)] & (n-@ \imath x) \\
@[\Phi\urcorner x \Psi x] \dashv \exists x(\forall y(\Psi y \leftrightarrow y=x) \wedge @[\Phi x]) & (w-@ \imath x)
\end{array}
$$

The two readings, obviously, are not equivalent. While the non-extensional sentential operator @ [...] is the main operator of the narrow scope reading, the wide scope reading amounts to an existentially quantified conjunction, only one conjunct of which is governed by @[...]. As a result, in (n-@1x) the definite description $\imath x \Psi x$ is embedded in a non-extensional context, whereas the matrix of $\imath x \Psi x$ is located in an extensional context in (w-@ $1 x)$.

In consequence, if the definite descriptions contained in the slingshot are systematically interpreted with wide scopes, the argument can be run even without @+ $@$-Subs. $\left(\zeta_{2}\right)$ exhibits the details of this 'wide scope slingshot',

\footnotetext{
${ }^{13}$ For more details on scope ambiguities involving definite descriptions cf. [38], $* 14$, [34], or [27, Chapter 4].
} 
where $F a, G a$, @[Fa], and $@[G a]$ again stand for (a), (b), (c), and (d), respectively.

\begin{tabular}{|c|c|c|c|}
\hline$\left(\zeta_{2}\right)$ & {$[1]$} & $F a$ & $\mathrm{~A}$ \\
\hline 2 & [2] & $G a$ & $\mathrm{~A}$ \\
\hline 3 & [3] & $@[F a]$ & A \\
\hline 2 & [4] & $a=\imath x(x=a \wedge G x)$ & 2, 1 -Conv \\
\hline 3 & {$[5]$} & $@[a=\imath x(x=a \wedge F x)]$ & 3 , @+ + -Conv \\
\hline 2 & {$[6]$} & $\begin{array}{l}\exists x(\forall y(y=a \wedge G y \leftrightarrow y=x) \wedge \\
\quad a=x)\end{array}$ & $4, * 14.01$ \\
\hline 3 & {$[7]$} & $\begin{array}{l}\exists x(\forall y(y=a \wedge F y \leftrightarrow y=x) \wedge \\
\quad @[a=x])\end{array}$ & $5, w-@ 1 x$ \\
\hline $8 *$ & {$[8]$} & $\begin{array}{l}\forall y(y=a \wedge G y \leftrightarrow y=b) \wedge \\
\quad a=b\end{array}$ & $\mathrm{~A}$ \\
\hline $9 *$ & [9] & $\begin{array}{l}\forall y(y=a \wedge F y \leftrightarrow y=c) \wedge \\
\quad @[a=c]\end{array}$ & A \\
\hline $9 *$ & {$[10]$} & $\begin{array}{l}(a=a \wedge F a \leftrightarrow a=c) \wedge \\
\quad @[a=c]\end{array}$ & $9, \forall$-elim \\
\hline $9 *$ & $\begin{array}{l}{[11]} \\
{[12]}\end{array}$ & $\begin{array}{l}a=a \wedge F a \leftrightarrow a=c \\
a=a\end{array}$ & $\begin{array}{l}\text { 10, ^-elim } \\
\top \text {-intro }\end{array}$ \\
\hline 1 & [13] & $a=a \wedge F a$ & $1,12 \wedge$-intro \\
\hline $1,9 *$ & [14] & $a=c$ & 11,13 MPP \\
\hline $8 *$ & {$[15]$} & $a=b$ & $8, \wedge$-elim \\
\hline $1,8 *, 9 *$ & [16] & $b=c$ & 14,15, trans of $=$ \\
\hline $1,8 *, 9 *$ & [17] & $\begin{array}{l}\forall y(y=a \wedge F y \leftrightarrow y=b) \wedge \\
\quad @[a=b]\end{array}$ & 9,16 PSST \\
\hline $1,8 *, 9 *$ & {$[18]$} & $@[a=b]$ & 17, ^-elim \\
\hline $8 *$ & [19] & $\forall y(y=a \wedge G y \leftrightarrow y=b)$ & $8, \wedge$-elim \\
\hline $1,8 *, 9 *$ & [20] & $\begin{array}{l}\forall y(y=a \wedge G y \leftrightarrow y=b) \wedge \\
\quad @[a=b]\end{array}$ & $18,19 \wedge$-intro \\
\hline $1,8 *, 9 *$ & {$[21]$} & $\begin{array}{l}\exists x(\forall y(y=a \wedge G y \leftrightarrow y=x) \wedge \\
\quad @[a=x])\end{array}$ & $20, \exists$-intro \\
\hline $1,2,9 *$ & {$[22]$} & $\begin{array}{l}\exists x(\forall y(y=a \wedge G y \leftrightarrow y=x) \wedge \\
\quad @[a=x])\end{array}$ & $6,8,21, \exists$-elim \\
\hline $1,2,3$ & {$[23]$} & $\begin{array}{l}\exists x(\forall y(y=a \wedge G y \leftrightarrow y=x) \wedge \\
\quad @[a=x])\end{array}$ & $7,9,22, \exists$-elim \\
\hline $1,2,3$ & {$[24]$} & $@[a=\imath x(x=a \wedge G x)]$ & 23,w-@ix \\
\hline $1,2,3$ & {$[25]$} & $@[G a]$ & 24, @+ - -CoNv \\
\hline
\end{tabular}

Contrary to $\left(\zeta_{1}\right)$, however, it is fairly obvious what the problem is with $\left(\zeta_{2}\right)$ : The transitions from line [3] to lines [5] and [7] and from [23] to lines [24] and [25] are not truth-preserving. In other words, if descriptions in factual contexts are read with wide scopes, @+ + -CoNv is not a correct inference rule. That means representatives of group $\mathcal{C}$ who endorse @ $+\imath$-Conv and reject $@+\imath$-SuBs based on a non-referential treatment of definite descriptions read definite descriptions in factual contexts with narrow scope. This then yields 
that @[...] is the main operator of the expression in line [5], which blocks the transition to [7] and prohibits subsequent applications of $\exists$-elimination. In sum, rejecting@+ @-SuBs on account of Russell's theory of descriptions and reading descriptions in factual contexts with narrow scope blocks the slingshot-an endorsement of @ +1 -CoNv and PSST for factual contexts notwithstanding.

This way around the slingshot enables group $\mathcal{C}$ to reject @ $+\imath$-SuBs while insisting on the correctness of PSST for factual contexts, and, thus, to draw significantly weaker consequences from the slingshot than their peers in group $\mathcal{B}$. Nonetheless, in what follows it shall be shown that even though a quantificational understanding of definite descriptions coupled with a narrow scope reading of descriptions in factual contexts allows for rebutting the slingshot while maintaining PSST, the unwanted consequences of such a rejection of @ + $\imath$-SuBS - to a large extent-are the same as the unwanted consequences of rejecting PSST for factual contexts: Not all informally valid arguments involving factual contexts can be formally reproduced. Consider the following argument:

4. (P1) The fact that the son of Servilia Caepionis stabbed Caesar caused the fact that Caesar died.

(P2) The son of Servilia Caepionis is identical to the husband of Porcia Catonis.

(C) The fact that the husband of Porcia Catonis stabbed Caesar caused the fact that Caesar died.

Causes and effects are entities in nature that are independent of our linguistic reference to them. It thus holds that if (P2) is true, $(\mathrm{P} 1)$ and $(\mathrm{C})$ state the same causal dependency as they pick out the same cause of the fact that Caesar died. Whenever (P1) and (P2) are true, so is (C). Plainly, the validity of 4 can easily be formally reproduced by means of @ $+\imath$-SuBs. However, if that rule is rejected based on a Russellian analysis of definite descriptions, formally capturing the validity of 4 gives rise to severe problems.

To see this, let us formalize (P1), (P2), and (C) in a Russellian vein. By letting $F$ stand for “. $\ldots$ is a son of Servilia Caepionis", $G$ for “. $\ldots$ stabbed Caesar", $p$ for "Caesar died" and by representing the factual causal connective "The fact that ... caused the fact that ..." by @[...,...], the narrow scope reading of $(\mathrm{P} 1)$ is expressible in terms of $\left(P_{1}^{n}\right)$ and the wide scope reading in terms of $\left(P_{1}^{w}\right) .^{14}$

$$
\begin{aligned}
& @[\exists x(\forall y(F y \leftrightarrow y=x) \wedge G x), p] \\
& \exists x(\forall y(F y \leftrightarrow y=x) \wedge @[G x, p])
\end{aligned}
$$

\footnotetext{
${ }^{14}$ The possibility of interpreting definite descriptions in factual causal statements to have wide scope is normally not considered in the literature on fact causation. I owe this point to Michael Gabbay.
} 
In contrast, there are no scope ambiguities involved in (P2). By introducing $H$ for "... is a husband of Porcia Catonis", a Russellian analysis of (P2) yields:

$$
\exists x \exists y\left(\forall z_{1}\left(F z_{1} \leftrightarrow z_{1}=x\right) \wedge \forall z_{2}\left(H z_{2} \leftrightarrow z_{2}=y\right) \wedge x=y\right)
$$

Analogously to the first premise, the conclusion of 4 allows for a narrow and a wide scope reading. They are formally reproduced in $\left(C^{n}\right)$ and $\left(C^{w}\right)$, respectively.

$$
\begin{aligned}
& @[\exists x(\forall y(H y \leftrightarrow y=x) \wedge G x), p] \\
& \exists x(\forall y(H y \leftrightarrow y=x) \wedge @[G x, p])
\end{aligned}
$$

$\left(P_{2}\right)$ states that the extensions of $F$ and $H$ comprise exactly one and the same element. As co-extensional predicates can only be substituted s.v. in extensional contexts, the validity of the two Russellian readings of 4 hinges on whether $F$ and $H$ occur in extensional contexts. While that is not the case for $\left(P_{1}^{n}\right)$ and $\left(C^{n}\right), F$ and $H$ are located outside of the context governed by the nonextensional causal connective in $\left(P_{1}^{w}\right)$ and $\left(C^{w}\right)$. Accordingly, $\left(P_{2}\right)$ licenses to replace $H$ for $F$ in $\left(P_{1}^{w}\right)$ which directly yields $\left(C^{w}\right)$. That is, whereas a narrow scope analysis of 4 generates an invalid formalization, the validity of 4 would be formally reproducible without the use of @ $+\imath$-Subs by drawing on a wide scope reading of the definite descriptions contained in (P1) and (C). Yet, as we have seen above, blocking the slingshot in the vein of group $\mathcal{C}$ presupposes definite descriptions embedded in factual contexts to have narrow scopes. As a direct consequence, endorsing @ $+\imath$-Conv and PSST for factual contexts, while rejecting @+ı-SuBS, renders it impossible to formally account for the validity of 4.

At this point, the representative of group $\mathcal{C}$ might be inclined to argue that definite descriptions in factual contexts must only be read with narrow scopes in connection with the slingshot argument but not in cases like 4 . More concretely, it might be held that only descriptive analyses of subjectpredicate statements as are generated by @+ı-CoNv, i.e. expressions of the form @ $[\alpha=\imath x(x=\alpha \wedge \Phi x)]$, must be systematically understood with narrow scope, while other descriptions embedded in factual contexts may be read with either narrow or wide scopes. However, as the Church-Quine-Davidson slingshot shows, descriptive analyses of this Gödelian type are not the only sort of expressions involving definite descriptions that can be used to run slingshot arguments. Hence, all other-potentially infinite-slingshot-inducing expressions would have to be confined to narrow scope interpretations as well. And what is the general criterion distinguishing those descriptions embedded in factual contexts that must be read with narrow scopes from those that merely may be thus interpreted?

Apart from problems of this kind, opting for the wide scope analysis of the descriptions contained in 4 receives no support from available semantics for factual causal statements, as are e.g. developed by Bennett [5] or Mellor [26]. According to these accounts, factual causal statements are of the form: "The fact that $\phi$ caused the fact that $\psi$ " is true iff $\Omega(\phi, \psi)$, where $\phi$ and $\psi$ stand for 
closed sentences and $\Omega$ denotes the set of conditions imposed on $\phi$ and $\psi$ by a pertaining theory in order for $\phi$ and $\psi$ to be causally connected. Common candidates for $\Omega$ are, for instance, that $\psi$ must be derivable from $\phi$ in combination with a causal law $\mathcal{L}$ and a causal background $B$ or that the probability/propensity/chance that $\psi$ holds is higher in the closest $\phi$-worlds than in the closest non- $\phi$-worlds. While the details of these different proposals for $\Omega$ are of no relevance for our purposes, it is crucial to note that factual causal statements relate logically independent closed sentences stating the causing and the caused facts. This constraint is straightforwardly met by the narrow scope readings of 4. $\left(P_{1}^{w}\right)$, for example, identifies the fact that there exists exactly one son of Servilia Caepionis who stabbed Caesar as cause of the fact that Caesar diedand analogously for $\left(C^{n}\right)$. The constraint, however, is violated in the wide scope readings. In $\left(P_{1}^{w}\right)$ and $\left(C^{w}\right)$ the first expression governed by $@[\ldots, \ldots]$ is an open sentence. Hence, the truth conditions of the third conjuncts within the scopes of the existential quantifiers of $\left(P_{1}^{w}\right)$ and $\left(C^{w}\right)$ are not determinable by means of available semantics for the factual causal connective.

Even if suitable semantics for factual causal contexts featuring Russellian descriptions with wide scopes should be developed in the future, it is highly dubitable that $\left(P_{1}^{w}\right)$ and $\left(C^{w}\right)$ can be said to capture the truth conditions of (P1) and (C), respectively. To see this, compare $\left(P_{1}^{w}\right)$ and (P1). If Servilia Caepionis, in fact, had two sons one of which was Brutus who stabbed Caesar, $\left(P_{1}^{w}\right)$ turns out false because its first conjunct is false. Yet, would we informally judge (P1) to be false as well? The answer to that question, I presume, depends on the preferred analysis of causation. The Humean, for instance, who wants to reduce causation to regularities or probability raising will say that (P1) is still true, because regularities may hold even if their antecedents are false or because the probability of Caesar dying is increased even if the description contained in (P1) is improper. In contrast, if causes are required to allow for deducing their effects in combination with relevant covering laws, (P1) will rather turn out false if its description is improper. Hence, the truth conditions of (P1) and (C), in the end, depend on the semantics of "The fact that ... caused the fact that ...". In order to formally reproduce this primacy of the factual causal connective for the truth conditions of $(\mathrm{P} 1)$ and $(\mathrm{C}), @[\ldots, \ldots]$ must be the main operator of corresponding formalizations. This condition is only satisfied by the narrow scope readings $\left(P_{1}^{n}\right)$ and $\left(C^{n}\right)$.

All in all, the slingshot conflict can be resolved either by drawing on a finegrained metaphysics of facts or on Russell's theory of descriptions. Both of these strategies, however, resolve it only at the price of giving rise to other conflicts between informal and formal validity assessments for arguments involving factual contexts. According to representatives of group $\mathcal{A}$, informally valid arguments as 1 and 3 cannot be formalized by formally valid inference schemes. The strategy of group $\mathcal{B}$, in turn, entails that informally valid arguments as 2, 3, and 4 cannot be formally mirrored. Finally, group $\mathcal{C}$ cannot formally account for the validity of arguments as 2 and 4.

Strategies to block the slingshot argument that cannot formally reproduce the validity of arguments as $1,2,3$, or 4 do not adequately capture our informal 
understanding of factual contexts. Members of groups $\mathcal{A}, \mathcal{B}$, and $\mathcal{C}$ either have to concede that their accounts are not complete insofar as they cannot reproduce all informally valid arguments involving factual contexts and thus violate (VP), or they are forced to revise informal validity assessments to the effect that not all of 1,2,3, and 4 are valid after all. Both of these consequences are unsatisfactory. The slingshot conflict, first and foremost, reveals that we do not yet fully understand how to apply available formal tools to factual contexts. A theory of adequate formalization for factual contexts must validate (VP) by reproducing our informal understanding of factual contexts, which, among other things, entails that $1,2,3$, and 4 are valid arguments and that the truth of (a) to (c) is compatible with the falsity of (d). Apparently, we informally attribute truth conditions to factual contexts such that logical equivalents and co-referring definite descriptions and proper names are substitutable s.v. in some factual contexts, while in others at least one of those substitutions is incorrect. The remainder of this paper is going to identify the subset of factual contexts for which both of these substitution principles hold.

\section{Revising @+१-SUBS}

Before we can tackle the question as to the details of a suitable restriction of @+ı-Conv or @+ı-Subs we, first, have to decide on which of the two rules to modify. On the face of it, there is no reason to expect that there is only one right way to go here. Formalisms are artificial products that build on conventional definitions whose usefulness must be assessed based on pragmatic criteria as simplicity or expressiveness. Accordingly, revising either@+ı-CoNv or @+ı-SuBs might eventually yield an adequate account of formalizing factual contexts. However, simplicity favors a revision of @+ $\imath$-SuBs, because the correctness of @ $+\imath$-SuBs is doubtful on grounds that are independent of the slingshot. Thus, since @ $+\imath$-SuBs must be modified anyway and since there are no comparable independent grounds to revise @ + there is no reason to also modify @ $+\imath$-Conv in light of the slingshot.

To see that restricting @ $+\imath$-SuBs is called for independently of the slingshot, consider the following statements: ${ }^{15}$

(e) Obama is identical to the one who is Obama and a politician.

(f) The fact that Obama is identical to the one who is Obama and a politician is identical to the fact that Obama is a politician.

(g) The fact that Obama is identical to Obama is identical to the fact that Obama is a politician.

Informally, we would say that (e) and (f) are true, while (g) is false. That is, (e) and (f) do not informally imply (g). Yet, if @ $+\imath$-SuBs were

${ }^{15} \mathrm{An}$ analogous example can be found in $[26,118]$. 
to hold unrestrictedly, the following would turn out to be a valid inference scheme-where $a$ refers to Obama, $F$ stands for "... is a politician", and @ [...] symbolizes the connective "The fact that ... is identical to the fact that Obama is a politician":

$$
\begin{array}{lllll}
\left(\zeta_{3}\right) & 1 & {[1]} & a=\imath x(x=a \wedge F x) & \mathrm{A} \\
& 2 & {[2]} & @[a=\imath x(x=a \wedge F x)] & \mathrm{A} \\
& 1,2 & {[3]} & @[a=a] & 1,2, @+\imath-\text { SuBS }
\end{array}
$$

While being a politician is a contingent fact, being self-identical is certainly not. Thus, these are two different facts about Obama. $\left(\zeta_{3}\right)$ demonstrates that @ + - -Subs alone may lead from truths to falsehoods. Similar transitions from true to false expressions may occur independently of Gödelian equivalents, for instance in case of what Quine [32, 148-149] has dubbed Kronecker descriptions:

$$
\begin{array}{lllll}
\left(\zeta_{4}\right) & 1 & {[1]} & 1=\imath x((x=1 \wedge F a) \vee(x=0 \wedge \neg F a)) & \mathrm{A} \\
& 2 & {[2]} & @[1=\imath x((x=1 \wedge F a) \vee(x=0 \wedge \neg F a))] & \mathrm{A} \\
1,2 & {[3]} & @[1=1] & 1,2, @+\imath-\text { SuBS }
\end{array}
$$

The expression in line [1] of $\left(\zeta_{4}\right)$ is formally equivalent to $\mathrm{Fa}$. In consequence, if @[...] is interpreted as above, line [2] is true, whereas line [3] is not. Hence, @ $+\imath$-Subs may turn true into false statements. These findings unmistakably show that @+ -SuBs is not correct as it was formulated in Section 2. We do not attribute truth conditions to factual contexts such that co-referring definite descriptions are generally substitutable s.v. @+ - -SuBs is not unrestrictedly applicable to factual contexts.

\section{Shallow Analysis}

@ + 1 -SuBs must be restricted in a way that, first, resolves the slingshot conflict by yielding the formal invalidity of the slingshot argument and of inferences as $\left(\zeta_{3}\right)$ and $\left(\zeta_{4}\right)$, and that, second, does not give rise to other conflicts between formal and informal validity assessments. As it turns out, the literature on adequate formalization provides all the necessary conceptual and criterial means required for such a restriction of @+ $@-$ SuBs. Those means just need to be assembled properly. To this end, we need to introduce the notions of correct formalization, structural complexity, and shallow formalization.

Authors developing criteria of adequate formalization, as Blau [7], Epstein $[15,16]$, Sainsbury [36], Brun [9], or Baumgartner and Lampert [4], all agree on one core criterion of adequate formalization: Adequate formalizations must be correct. Concisely put, correctness amounts to the following: The formalization $\Gamma_{1}$ of a statement $A_{1}$ is correct iff whatever formally follows from $\Gamma_{1}$ is a formalization of a natural language statement that informally follows from $A_{1}$, and whatever formally implies $\Gamma_{1}$ is a formalization of a natural language statement that informally implies $A_{1}$. Correctness is necessary but not sufficient for the adequacy of formalizations. Formalization theories complement it with 
further criteria, most of which are of no relevance to our current purposes. Such as to illustrate the application of the correctness criterion, consider statement (h) along with the forthright formalization Eq. 2.

(h) Obama is a politician or Obama is married.

$$
p \vee q
$$

$p$ : Obama is a politician.; $q$ : Obama is married.

To establish the correctness of Eq. 2 for (h) it must be shown that Eq. 2 does not allow for inferences that (h) does not sanction and that Eq. 2 does not follow from premises $(\mathrm{h})$ does not informally follow from. For example, Eq. 2 follows from $p$ and from $q$. In accordance, (h) informally follows from "Obama is a politician" and from "Obama is married". Or Eq. 2 formally implies $p \vee q \vee r$ and $\neg p \rightarrow q$. (h) correspondingly informally entails "Obama is a politician or Obama is married or Shamus is Pope" as well as "If Obama is not a politician, Obama is married". The correctness of Eq. 2 for $(\mathrm{h})$ is proven if it is established that all formal dependencies in which Eq. 2 is involved have informal counterparts. ${ }^{16}$ Plainly, any formula that is logically equivalent to Eq. 2 is involved in the exact same inferential dependencies as Eq. 2. Therefore, expressions as $p \vee(q \wedge r \leftrightarrow r)$ or $(r \wedge \neg r) \vee(p \vee q)$-or any other equivalent formula-are just as correct for (h) as Eq. 2. Moreover, of course, (h) can also be correctly formalized by first-order means:

$$
\begin{gathered}
F a \vee G a \\
a=\imath x(x=a \wedge(F x \vee G x)) \\
1=\imath x((x=1 \wedge(F a \vee G a)) \vee(x=0 \wedge \neg(F a \vee G a)) \\
a \text { : Obama } ; F: \ldots \text { is a politician ; } G \text { : ... is married }
\end{gathered}
$$

While Eqs. 3, 4, and 5 are formally equivalent, they are not formally equivalent to Eq. 2. That is, there may exist numerous non-equivalent correct formalizations of one particular statement $A_{1}$. To every correct formalization of $A_{1}$ there exists an infinite amount of equivalent correct formalizations. The correct formalizations of a statement can thus be grouped in equivalence classes $\varphi_{1}, \varphi_{2}, \ldots, \varphi_{n}$.

The formalizations contained in such equivalence classes can be ordered with respect to structural complexity. For instance, Eq. 5 introduces more structural complexity than Eq. 4, which, in turn, is more complex than Eq. 3. As Hodges [21, 47] puts it, a complexity measure numerically reproduces the property that "any formula has a higher complexity than any of its proper subformulas". Accordingly, he proposes to define the structural complexity of a formula $\Gamma_{1}$ to be the number of its subformulas, where the subformulas of $\Gamma_{1}$ are the atomic expressions contained in $\Gamma_{1}$, the molecular expressions in $\Gamma_{1}$

\footnotetext{
${ }^{16}$ For more details on correctness and the application of this criterion cf. [9] or [4].
} 
composed by means of logical connectives and quantifiers, and $\Gamma_{1}$ itself. ${ }^{17}$ For instance, Eq. 3 has complexity 3, for it contains the following subformulas: $F a$, $G a$, and $F a \vee G a$. By contrast, Eq. 4 has complexity 6, as it is composed of $F x$, $G x, x=a, F x \vee G x, x=a \wedge(F x \vee G x)$, and $a=\imath x(x=a \wedge(F x \vee G x))$.

Finally, Quine $[32,160]$ provides a maxim of adequate formalizationintended to complement correctness- that will prove to be of crucial importance for the formalization of factual contexts, viz. the maxim of shallow analysis: "expose no more logical structure than seems useful for the deduction or other inquiry at hand". ${ }^{18}$ The maxim stipulates that formalizations should only introduce as much structure as is needed to fulfill the purposes pursued by resorting to formalizations in the first place. It determines to avoid structural complexity that is redundant. Or differently, it requires to always choose the least complex correct formalization that serves the purposes at hand. To illustrate, consider the informally valid argument 5 and assume the purpose of formalizing 5 is to formally account for its validity:

5. Socrates is wise. Wisdom is a virtue. Therefore, Socrates is virtuous.

A correct formalization of 5 that reproduces its validity is the formally valid inference scheme Eq. 6.

$$
\begin{gathered}
F a, \forall x(F x \rightarrow G x) \vdash G a \\
F: \ldots \text { is wise } ; G: \ldots \text { is virtuous } ; a: \text { Socrates }
\end{gathered}
$$

Of course, the validity of 5 could also be formally reproduced by introducing additional structure as done in Eqs. 7 and 8, which are to be read relative to the interpretation given in Eq. 6 as well.

$$
\begin{gathered}
F a \wedge a=a, \forall x(F x \wedge x=x \rightarrow G x) \vdash G a \\
a=\imath x(x=a \wedge F x), \forall x(F x \rightarrow G x) \vdash a=\imath x(x=a \wedge G x)
\end{gathered}
$$

The premises and conclusion of Eqs. 7 and 8 are each formally equivalent to their respective counterparts in Eq. 6. Yet, even though Eqs. 7 and 8 are, hence, correct for 5 iff Eq. 6 is correct for 5, Eqs. 7 and 8 violate shallowness, for they involve redundant logical structure. There exists another correct formalization of 5 which is less complex than Eqs. 7 and 8 and which also accounts for the validity of 5, viz. Eq. 6. Among the correct formalizations of a statement $A_{1}$ that differ with respect to structural complexity and that fulfill the purposes at hand only one with lowest complexity measure constitutes a

\footnotetext{
${ }^{17}$ For more details cf. [21, 46-47] or [16, 55-56]. Other complexity measures have been proposed in the literature, cf. e.g. [22, 62] or [9, Chapters 13.4-13.6].

${ }^{18}$ Cf. also [33, 198], [20, 243], or [9, 322].
} 
shallow formalization of $A_{1}$. The only purpose of formalizing a statement $A_{1}$ that is of interest in this paper is the formal reproduction of the validity or invalidity of the arguments that $A_{1}$ is part of. In extensional contexts, equivalent formalizations of a statement $A_{1}$ cannot differ with respect to accounting for the (in)validity of arguments comprising $A_{1}$. If $A_{1}, A_{2}, \ldots, A_{m}$ informally entail $A_{n}$ and this dependence is reproduced by the correct formalizations contained in the inference scheme $\Gamma_{1}, \Gamma_{2}, \ldots, \Gamma_{m} \vdash \Gamma_{n}$, replacing any $\Gamma_{i}, 1 \leq$ $i \leq n$, by a formally equivalent expression $\Gamma_{i}^{*}$ with higher complexity measure than $\Gamma_{i}$ does not affect the formal validity of that inference scheme, and hence equally reproduces the corresponding informal dependence-and analogously for informal independencies. That is, of a set $\varphi_{1}$ of formally equivalent formalizations that are correct for $A_{1}$ and contribute to accounting for the (in)validity of $A_{1}$ 's argumentative context, only the least complex satisfy shallowness. For the purposes of this paper we can thus define the notion of a shallow formalization as follows: A formalization $\Gamma_{1}$ of a statement $A_{1}$ is shallow iff $\Gamma_{1}$ is correct for $A_{1}$ and there does not exist another formalization $\Gamma_{1}^{*}$ of $A_{1}$ that is formally equivalent to $\Gamma_{1}$ and has a lower complexity measure than $\Gamma_{1}$.

Introducing redundant structure, i.e. additional structural complexity that does not change a subformula's truth conditions, into extensional contexts merely generates unnecessary complications, but does not turn valid inference schemes into invalid ones, or vice versa. Thus, Quine's primary rationale for the maxim of shallow analysis was simply to avoid superfluous work in the course of formalizing extensional statements. ${ }^{19}$ However, what holds for extensional contexts does not necessarily hold for non-extensional ones as well. Indeed, the slingshot argument reveals that replacing a first-order expression as $F a$ within a factual context by a formally equivalent, yet more complex expression as $a=\imath x(x=a \wedge F x)$ may give rise to incorrect inferences, i.e. to inferences that do not have informal counterparts. Moreover, these incorrect inferences could not be drawn on the mere basis of $F a$. While in extensional contexts replacing a formalization $\Gamma_{i}$ by a more complex equivalent expression $\Gamma_{i}^{*}$ cannot induce incorrect inferences, factual contexts are sensitive to the introduction of excessive logical structure. That is, if a correct formalization $\Gamma_{1}$ of $A_{1}$ that contributes to formally reproducing the (in)validity of an argument featuring "The fact that $A_{1}$ is ..." is replaced by an equivalent more complex formalization $\Gamma_{1}^{*}$, it may turn out that the (in)validity of the corresponding argument is no longer successfully accounted for. In consequence, the status of shallow analysis in factual contexts differs significantly from its status in extensional contexts. In factual contexts, excessive logical structure does not merely induce unnecessary complications, but, as the slingshot demonstrates, may give rise to incorrect inferences and to conflicts between informal and formal validity assessments.

\footnotetext{
${ }^{19}$ Correspondingly, shallow analysis is commonly not seen as a necessary condition of adequate formalization (cf. [9, Chapter 13]).
} 
These considerations and the conceptual resources assembled above pave the way towards a restriction of @+ $@-$ SuBs that renders $\left(\zeta_{1}\right),\left(\zeta_{3}\right)$, and $\left(\zeta_{4}\right)$ formally invalid and allows for the formal reproduction of the validity of arguments 1 to 5: Within factual contexts, co-referring definite descriptions and proper names can only be replaced s.v. in shallow formalizations. More specifically, @+ -SuBs must be restricted such that, first, it is only applicable to shallow formalizations embedded in factual contexts, i.e. to shallow factual contexts, and second, such that no redundant structure is introduced, that is, such that shallowness is preserved. If we let $\Sigma_{s}(\alpha)$ and $\Sigma_{S}(\imath x \Phi x)$ stand for shallow formalizations containing the name $\alpha$ and the description $\imath x \Phi x$, respectively, the required restriction of @ $+\imath$-Subs can be spelled out as follows:

$$
\begin{array}{llll}
@_{s}+1 \text {-SuBS : } & \imath x \Phi x=\alpha & \alpha=\imath x \Phi x & \imath x \Phi x=\imath x \Psi x \\
& \frac{@\left[\Sigma_{s}(\imath x \Phi x)\right]}{@\left[\Sigma_{s}(\alpha)\right]} & \frac{@\left[\Sigma_{s}(\alpha)\right]}{@\left[\Sigma_{s}(\imath x \Phi x)\right]} & \frac{@\left[\Sigma_{s}(\imath x \Phi x)\right]}{@\left[\Sigma_{S}(\imath x \Psi x)\right]}
\end{array}
$$

As shall be substantiated in the following, @ ${ }_{s}+\imath$-SuBs resolves the slingshot conflict without giving rise to other conflicts between formal and informal validity assessments.

To see that the Gödelian slingshot argument turns out to be invalid, given that @+ + -SuBs is rejected in favor of $@_{s}+\imath$-SuBs, reconsider $\left(\zeta_{1}\right)$. This exemplary instance of the slingshot involves the statements (a), (b), (c), and (d), the first three of which do not informally imply the fourth. That is, "(a), (b), (c). Therefore, (d)" is an informally invalid argument. This invalidity can be formally accounted for in a straightforward way:

$$
F a, G a, @[F a] \nvdash @[G a]
$$

$a$ : Obama ; $F: \ldots$ is a politician ; $G: \ldots$ is married

$@[. .$.$] : The fact that ... is identical to the fact that Obama is a politician.$

Equation 9 comprises correct and minimally complex formalizations of (a) to (d). There do not exist formally equivalent expressions with lower complexity measures, for the complexity measures of $F a, G a$ and of @ $[F a], @[G a]$ are minimal: 1 and 2, respectively. That is, Eq. 9 features shallow formalizations. As there are no identity statements, it is impossible to apply @ ${ }_{s}+\imath$-SuBs, and hence impossible to deduce @ [Ga] from $F a, G a$, and @ $[F a]$.

As we have seen in Section 2 though, 1-Conv and@+ı-Conv permit to introduce identity statements into the formalizations of (a) and (c). However, since $a=\imath x(x=a \wedge F x)$ is formally equivalent to $F a$, its additional complexity is redundant. $a=\imath x(x=a \wedge F x)$ is on a par with infinitely many other formulas that are formally equivalent to $F a$, as $F a \wedge a=a$ or $1=\imath x((x=1 \wedge F a) \vee$ $(x=0 \wedge \neg F a)$ ), all of which feature excessive logical structure. None of these formalizations is shallow. Accordingly, $@_{s}+\imath$-Subs cannot be applied if any of them is embedded in a factual context. This, in turn, renders the step from line [7] to [8] of $\left(\zeta_{1}\right)$ invalid, and thereby blocks the slingshot. The slingshot 
argument as given in $\left(\zeta_{1}\right)$ essentially draws on the introduction of logical structure that in no way affects the truth conditions of the formulas embedded in the corresponding factual contexts. While in extensional contexts such excessive structure is harmless, in factual contexts it may give rise to conflicts between formal and informal validity assessments. Therefore, to adequately formally reproduce inferential dependencies among (or truth conditions of) factual statements, the substitutability of singular terms must be restricted to shallow formalizations.

Such a restriction of @ $+\imath$-Subs also invalidates $\left(\zeta_{3}\right)$. The statement (e), i.e. "Obama is identical to the one who is Obama and a politician" is not shallowly formalized in terms of $a=x x(x=a \wedge F x)$, with $F$ representing "... is a politician" and $a$ referring to Obama. There exists a formally equivalent correct formalization with lower complexity measure, viz. Fa. Notwithstanding its misleading grammatical surface, (e) is informally equivalent to "Obama is a politician". Hence, both of the equivalent formalizations $a=\imath x(x=a \wedge F x)$ and $F a$ are correct for (e) as well as for "Obama is a politician". The formalization with lowest complexity measure, i.e. the shallow formalization of both statements, is $F a$. Therefore, $@_{s}+\imath$-Subs does not sanction the transition of lines [1] and [2] of $\left(\zeta_{3}\right)$ to line [3]. For analogous reasons, rejecting @ $+\imath$-SuBS in favor of $@_{s}+\imath$-SuBs renders $\left(\zeta_{4}\right)$ invalid as well.

While @ ${ }_{s}+\imath$-SuBs blocks both the slingshot and incorrect inferences along the lines of $\left(\zeta_{3}\right)$ and $\left(\zeta_{4}\right)$, direct applications of $@_{s}+\imath$-SuBs to shallow formalizations of the statements involved in arguments as 2,3 , or 4 straightforwardly account for their informal validity. To see this, consider argument 4 and let $F$ stand for "... is a son of Servilia Caepionis", $G$ for "... stabbed Caesar", $H$ for "... is a husband of Porcia Catonis", $p$ for "Caesar died", and @ $[\ldots, \ldots]$ for the factual causal connective. The following application of $@_{s}+1$-Subs directly leads from the premises of 4 to its conclusion:

$\begin{array}{lllll}\left(\zeta_{5}\right) & 1 & {[1]} & @[G x F x, p] & \mathrm{A} \\ & 2 & {[2]} & \imath x F x=\imath x H x & \mathrm{~A} \\ & 1,2 & {[3]} & @[G x H x, p] & 1,2, @_{s}+\imath \text {-SUBS }\end{array}$

Similarly, if we additionally introduce $a$ as referring to Brutus, the informal validity of 2 is reproducible by means of $@_{s}+\imath$-SuBs:

$\begin{array}{lllll}\left(\zeta_{6}\right) & 1 & {[1]} & @[G a, p] & \mathrm{A} \\ & 2 & {[2]} & a=\imath x F x & \mathrm{~A} \\ & 1,2 & {[3]} & @[G i x F x, p] & 1,2, @_{s}+\imath \text {-SUBS }\end{array}$

Note that $@_{s}+\imath$-SuBs renders the formal validity of inference schemes involving factual contexts dependent on their structural complexity. In this respect factual and extensional contexts differ significantly: While the introduction of redundant structure into a valid inference scheme only featuring extensional contexts cannot overturn the latter's validity, such specifications of inference schemes containing factual contexts do not necessarily conserve validity. In consequence, to formally reproduce the informal validity of a purely extensional argument $S_{1}, S_{1}$ may be formalized at any level of complexity, 
whereas to formally reproduce the informal validity of a factual argument $S_{2}$, $S_{2}$ must be formalized shallowly. Consider the following variation of 2 :

6. The fact that Brutus is the one who is Brutus and stabbed Caesar caused the fact that Caesar died. Brutus is identical to the son of Servilia Caepionis. Therefore, the fact that the son of Servilia Caepionis is the one who is the son of Servilia Caepionis and stabbed Caesar caused the fact that Caesar died.

Just as 2, 6 is an informally valid argument. Indeed, the premises and conclusion of 2 and 6 are informally equivalent — the component statements of the latter being unnecessarily cumbersome. Yet, only a shallow formalization that correctly reproduces the premises and conclusion of 6 as Eq. 10-whose formal validity is proven in $\left(\zeta_{6}\right)$-mirrors the validity of 6 . By contrast, formalization Eq. 11 which correctly but non-shallowly captures the premises and conclusion of 6 amounts to an invalid inference scheme, because @ ${ }_{s}+\imath$-SuBs is not applicable to the first premise of Eq. 11.

$$
\begin{gathered}
@[G a, p], a=\imath x F x \vdash @[G \imath x F x, p] \\
@[a=\imath x(x=a \wedge G x), p], a=\imath y F y \nvdash @[\imath y F y=\imath x(x=\imath y F y \wedge G x), p]
\end{gathered}
$$

That means the grammatical surface of argument 6 is misleading as to the valid form 6 instantiates. To formally mirror the validity of informally valid arguments featuring factual contexts, these arguments must be formalized shallowly-even if that requires digressing from the grammatical surface of formalized arguments.

In sum, an adequate formal reproduction of factual contexts calls for strict adherence to Quine's maxim of shallow analysis. By shallowly formalizing factual contexts the slingshot argument is blocked, while the validity of arguments as 1 to 4 and 6 remains formally reproducible.

\section{Conclusion}

The slingshot argument essentially draws on the introduction of redundant logical structure into factual contexts. While such redundancies do not give rise to incorrect inferences in extensional contexts, factual contexts are sensitive to excessive logical structure. That is the primary lesson to draw from the slingshot argument. This paper has moreover shown that factual contexts differ from hyperintensional ones as belief contexts in two crucial respects: First, logical equivalents can be substituted s.v. within factual contexts while such substitutions are invalid in hyperintensional contexts, and second, a specific type of expressions, viz. shallow ones, allow for substitutions of co-referring singular terms within factual contexts while such substitutions are generally invalid within hyperintensional ones. In this sense, factual contexts occupy a middle ground between extensional and hyperintensional contexts. 
Thus, this paper suggests the following answers to our introductory questions $\left(Q_{1}\right)$ and $\left(Q_{2}\right)$.

$\left(A_{1}\right)$ If definite descriptions embedded in factual contexts are interpreted referentially, logical equivalents can generally be substituted s.v. within factual contexts, i.e. PSLE and its special case 1 -Conv hold unrestrictedly for factual contexts. If definite descriptions in factual contexts are spelled out in a Russellian vein, @+ -Conv only holds if definite descriptions are interpreted with narrow scopes. Furthermore, coreferring proper names and definite descriptions can be substituted s.v. in shallow factual contexts. That is, @ $+\imath$-SuBs must be restricted in terms of $@_{s}+\imath$-SuBs.

$\left(A_{2}\right)$ Adequate formalizations of factual contexts must not only be correct but moreover shallow.

Of course, $\left(A_{1}\right)$ and $\left(A_{2}\right)$ do not yet constitute an exhaustive account of formalizing factual contexts. Many open questions remain. For instance, correctness and shallowness are necessary but certainly not sufficient for the adequacy of formalizations of factual contexts, or PSLE and $@_{s}+\imath$-SuBS are not the only inference rules that can be validly applied to factual contexts. However, $\left(A_{1}\right)$ and $\left(A_{2}\right)$ constitute a necessary core of an adequate account of formalizing factual contexts which avoids conflicts between assessments of informal and formal dependencies among statements featuring factual contexts, and thus validates (VP). We have found that such conflicts arise because formal systems that treat definite descriptions or class abstracts as primitive referring terms allow for the introduction of certain kinds of excessive logical structure into factual contexts to which these contexts are sensitive. If factual contexts are consistently shallowly formalized, such conflicts are avoided. This finding does not teach us anything about the metaphysics of facts, which, after all, must be conceived of in light of the theoretical work facts are supposed to do within a broader metaphysical (or philosophical) picture. Nor does it teach us anything about the quality of formalisms featuring primitive iota-expressions or class abstracts, which, after all, have proven to be very profitably applicable, for instance, to the formalization of statements of number theory (cf. e.g. [6]). Rather, this finding teaches us something about how our informal understanding of factual contexts is adequately reproduced by means of available formal tools. If Quine's maxim of shallow analysis is rigorously respected in the course of formalizing factual contexts, definite descriptions can be understood referentially without conflicts arising between informal and formal validity assessments with respect to arguments featuring factual contexts, and, accordingly, without having to settle for a fine-grained metaphysics of facts.

Acknowledgements I am particularly grateful to Timm Lampert for countless discussions about facts, slingshots, and logical formalization. Moreover, I thank Delphine Chapuis-Schmitz, Sebastian Leugger, Jonas Rogger, and the anonymous referees for this journal for helpful 
comments on an earlier draft. Finally, I am indebted to the Swiss National Science Foundation for generous support of this work (grant PP001-114812/1).

\section{References}

1. Anscombe, G. E. M. (1969). Causality and extensionality. Journal of Philosophy, 66, 152-159.

2. Barwise, J., \& Perry, J. (1996). Semantic innocence and uncompromising situations. In A. P. Martinich (Ed.), The philosophy of language (pp. 369-381). Oxford: Oxford University Press (originally published 1975 in Midwest Studies in Philosophy).

3. Baumgartner, M. (forthcoming). Informal reasoning and logical formalization. In S. Conrad \& S. Imhof (Eds.), Ding und Begriff. Frankfurt a.M.: Ontos.

4. Baumgartner, M., \& Lampert, T. (2008). Adequate formalization. Synthese, 164, 93-115.

5. Bennett, J. (1988). Events and their names. Indianapolis: Hackett.

6. Bernays, P. (1958). Axiomatic set theory. Amsterdam: North-Holland.

7. Blau, U. (1977). Die dreiwertige Logik der Sprache. Berlin: de Gruyter.

8. Brandl, J. (1991). Some remarks on the "slingshot" argument. In G. Dorn \& G. Schurz (Eds.), Advances of scientific philosophy (pp. 421-437). Amsterdam: Rodopi.

9. Brun, G. (2004). Die richtige Formel. Philosophische Probleme der logischen Formalisierung. Frankfurt a.M.: Ontos.

10. Carnap, R. (1947). Meaning and necessity. Chicago: University of Chicago Press.

11. Church, A. (1943). Review of Carnap's Introduction to semantics. Philosophical Review, 52, 298-304.

12. Cummins, R., \& Gottlieb, D. (1972). On an argument for truth-functionality. American Philosophical Quarterly, 9, 265-269.

13. Davidson, D. (1980). Essays on actions and events. Oxford: Clarendon.

14. Drai, D. (2002). The slingshot argument: An improved version. Ratio, 15, 194-204.

15. Epstein, R. L. (1990). The semantic foundations of logic: Propositional logic. Dordrecht: Kluwer.

16. Epstein, R. L. (1994). The semantic foundations of logic: Predicate logic. Oxford: Oxford University Press.

17. Etchemendy, J. (1990). The concept of logical consequence. Cambridge: Harvard University Press.

18. Frege, G. (1892/1984). On sense and meaning. In B. McGuinness (Ed.), Collected papers on mathematics, logic, and philosophy. Oxford: Basil Blackwell.

19. Gödel, K. (1944). Russell's mathematical logic. In P. A. Schilpp (Ed.), The philosophy of Betrand Russell (pp. 123-153). New York: Tudor.

20. Haack, R. J. (1978). Quine's theory of logic. Erkenntnis, 13, 231-259.

21. Hodges, W. (2001). Elementary predicate logic. In D. M. Gabbay \& F. Guenthner (Eds.), Handbook of philosophical logic (2nd Ed., Vol. 1, pp. 1-130). Dordrecht: Kluwer Academic.

22. Iacona, A. (2004). Modal predicates. Australasian Journal of Logic, 2, 56-69.

23. Koons, R. C. (2000). Realism regained. An exact theory of causation, teleology, and the mind. Oxford: Oxford University Press.

24. Lampert, T., \& Baumgartner, M. (2010). The problem of validity proofs. Grazer Philosophische Studien, 80, 79-109.

25. Mellor, D. H. (1991). The singularity affecting facts of causation. In Matters of metaphysics (pp. 201-224). Cambridge: Cambridge University Press.

26. Mellor, D. H. (1995). The facts of causation. London: Routledge.

27. Neale, S. (1990). Descriptions. Cambridge: MIT Press.

28. Neale, S. (1995). The philosophical significance of Gödel's slingshot. Mind, 104, 761-825.

29. Neale, S. (2001). Facing facts. Oxford: Clarendon.

30. Oliver, J. W. (1967). Formal fallacies and other invalid arguments. Mind, 76, 463-478.

31. Quine, W. v. O. (1953/1975). Three grades of modal involvement. In The ways of paradox (pp. 158-176). Cambridge: Harvard University Press.

32. Quine, W. v. O. (1960). Word and object. Cambridge: MIT Press.

33. Quine, W. v. O. (1982). Methods of logic, 4th Ed. Cambridge: Harvard University Press. 
34. Russell, B. (1905). On denoting. Mind, 14, 479-493.

35. Russell, B. (1977). The philosophy of logical atomism. In R. Marsh (Ed.), Logic and knowledge (pp. 175-281). New York: Allen \& Unwin.

36. Sainsbury, M. (2001). Logical forms. An introduction to philosophical logic, 2nd Ed. Malden, MA: Blackwell.

37. Sobel, J. H. (2008). Collapsing arguments for facts and propositions. Australasian Journal of Logic, 6, 122-161.

38. Whitehead, A. N., \& Russell, B. (1910). Principia mathematica. Cambridge: Cambridge University Press.

39. Williamson, J. (1976). Facts and truth. The Philosophical Quarterly, 26, 203-216. 